\title{
DIFICULTADES PARA LA CONSTRUCCIÓN DE LA LITERACIDAD EN LA EDUCACIÓN MÉDICA
}

DIFFICULTIES FOR THE CONSTRUCTION OF LITERACITY IN MEDICAL EDUCATION

Volumen 13, Número 2 Mayo - Agosto

pp. 1-18

Este número se publicó el 30 de mayo de 2013

\author{
Juan Manuel Muñoz Cano \\ Teresita Maldonado Salazar
}

Revista indizada en REDALYC, $\underline{\text { SCIELO }}$

Revista distribuida en las bases de datos:

CATÁLOGO DE LATINDEX, IRESIE, CLASE, DIALNET, DOAJ, E-REVIST@S, SHERPA/ROMEO, QUALIS, MIAR

Revista registrada en los directorios:

ULRICH'S $, \underline{R E D I E}, \underline{R I N A C E}, \underline{\text { OEI }}$ MAESTROTECA, PREAL, $\underline{\text { CLASCO }}$ 


\title{
DIFICULTADES PARA LA CONSTRUCCIÓN DE LA LITERACIDAD EN LA EDUCACIÓN MÉDICA \\ DIFFICULTIES FOR THE CONSTRUCTION OF LITERACITY IN MEDICAL EDUCATION
}

\author{
Juan Manuel Muñoz Cano ${ }^{1}$ \\ Teresita Maldonado Salazar ${ }^{2}$
}

\begin{abstract}
Resumen: El objetivo del estudio que se describe en este artículo fue analizar los apoyos pedagógicos con que contaron estudiantes de medicina para construir conocimiento a partir de información que ha de leerse en diferentes textos y en diferentes formatos. Para ello, se realizó una investigación cualitativa de estudio de caso de tipo de correlación/prospectivo. Los participantes fueron 100 de los 350 estudiantes de nuevo ingreso a Medicina en la Universidad de Tabasco, México, para el ciclo escolar que inició en la última semana de agosto de 2011. Las categorías de análisis fueron: a) las indicaciones y apoyos que recibieron de los docentes para la elaboración de escritos, b) las prácticas del estudiantado para hacer sus escritos y c) las motivaciones que tenían los estudiantes para desarrollarlos. Se encontró que el personal docente, en la mayor parte de las asignaturas, les solicitó escritos de tipo ensayo. Las instrucciones de docentes fueron con predominio de formato (extensión, tipo de letra). Los estudiantes y las estudiantes valoraron la escritura académica y la consideran necesaria para aprender y elaborar opiniones informadas, pero los escritos los calificaron prioritariamente los docentes con base en ortografía y gramática. Es necesaria la retroalimentación presencial y virtual para el logro de la competencia de literacidad.
\end{abstract}

Palabras clave: ALFABETIZACIÓN, EDUCACIÓN MÉDICA, GESTIÓN DEL CONOCIMIENTO, LENGUAJE ESCRITO, PRODUCCIÓN DE TEXTOS, MÉXICO

\begin{abstract}
The purpose of the study described in this article was to analyze the pedagogical supports that have medical students to construct knowledge from information that has to be read in different texts in different formats. It was a qualitative case study of correlation type research / prospective. The participants were 100 of 350 students from new entrants to medicine at the University of Tabasco, Mexico, for the school year that began in the last week of August, 2011. The analysis categories were a) indications and support they received of teachers for the preparation of briefs, b) the practices of the students to make their writings and c) motivations that students had to develop them. The instructions of teachers were predominantly about format (extension, font). Students valued academic writing and considered it necessary to learn and to develop informed opinions, but most teachers rated the writings based on spelling and grammar. It is necessary to have online and presence feedback for the achievement of the literacity competence.
\end{abstract}

Keywords: ALPHABETIZATION, MEDICAL EDUCATION, MANAGEMENT OF THE KNOWLEDGE, WRITTEN LANGUAGE, TEXT PRODUCTION, MEXICO

\footnotetext{
${ }^{1}$ Docente en la escuela de Medicina de la Universidad Juárez Autónoma de Tabasco, México. Médico, Maestro en Ciencias en Biomedicina Molecular. Dirección electrónica: juan.munoz@ujat.mx

${ }^{2}$ Docente en la Universidad Pedagógica Nacional de México. Profesora de Educación Preescolar, Licenciada en Pedagogía, Maestra en Educación Ambiental. Dirección electrónica: tmaldonado@semarnat.gob.mx
}

Artículo recibido: 22 de noviembre, 2012

Aprobado: 20 de mayo, 2013 


\section{Introducción}

La literacidad se define como el "conjunto de conocimientos, valores y comportamientos implicados en el ejercicio de las prácticas letradas de comprensión y producción" (Cassany y Morales, 2008, p. 69). También, es "el conjunto de competencias que hacen hábil a una persona para producir, gestionar, editar, recibir y analizar información en un determinado contexto para transformarla en conocimiento" (Caro y Arbeláez, 2009, p.5). Esta habilidad se encuentra explícita entre las competencias necesarias del médico en servicio (como capacidad o experticia en un dominio) de la Asociación Mexicana de Escuelas y Facultades de Medicina (AMFEM), pues son condición para:

Utilizar el conocimiento teórico, científico, epidemiológico y clínico con la finalidad de tomar decisiones médicas, a fin de elaborar modelos que permitan promover la salud, disminuir riesgos, limitar los daños y proponer abordajes viables a los problemas de salud, adecuándose a las necesidades y condiciones de los pacientes, la comunidad y sociedad. (Abreu et al., 2008, p. 12)

Ello implica desarrollar procesos educativos para que el estudiantado transite hacia la búsqueda, identificación, análisis y apropiación de la información —sobre todo de la Internet- como medio de difusión, así como hacia la construcción de conceptos médicos como nivel superior de tratamiento de esa información (Caro y Arbeláez, 2009, p. 4); sin embargo, de manera habitual no se fomentan el pensamiento sistémico ni el pensamiento complejo para el desempeño profesional sustentado en una medicina que considere las dimensiones biológica, psicológica, social y cultural de la localidad donde se ejerce, pues estos ámbitos determinan las necesidades de información que para cada comunidad se necesita en la resolución de problemas, tanto los particulares a esa comunidad (por ejemplo: enfermedad de Chagas) como los generales de un mundo globalizado (por ejemplo: diabetes mellitus de tipo 2).

En las prácticas educativas tradicionales —donde el énfasis está en el estudio de cada uno de los temas de una disciplina, independientemente de cómo o cuándo pueden ser aplicados (Díaz, 2005) —los docentes no facilitan el desarrollo de habilidades de lectura y escritura como medio para permitir la construcción de conocimiento. Esta falta de capacidades se ha documentado en las universidades del continente americano (Bitram et al., 2009; Caldera, 2007; Fregoso, 2008; Hernández, 2000; Morales, 2002; Peña, 2008; 
Pérez, 2008), y explica deficiencias en la atención médica en el nivel global (Centres for Disease Control and Prevention [CDC], s.f.; Institute of Medicine of the National Academies [IMNA], 2012; Starfield, 2000; Warner et al., 2009) lo que hace necesario la modificación de los procesos educativos (General Medical Council [GMC], 2009; Organización Mundial de la Salud [OMS], 2008; United Nations Educational, Scientific and Cultural Organization [UNESCO], 2009). En paralelo, se ha generado el sustento de propuestas de ambientes de aprendizaje con base en hallazgos científicos con el propósito de que los estudiantes "piensen y lean críticamente y se expresen clara y convincentemente" (Committee on Developments in the Science of Learning [CDSL], 2000, capítulo 1), por lo que es imperativo se pongan en marcha escenarios educativos para superar el modelo tradicional.

\subsection{La literacidad como competencia transversal en educación superior}

Si "la práctica de la lectura" debe desarrollar desde la escuela de nivel básico "la capacidad de observación, atención, concentración, análisis y espíritu crítico, además de generar reflexión y diálogo" (Secretaría de Educación Pública [SEP], 2011, p. 87), es incongruente que sólo mediante el énfasis en el número de palabras que los estudiantes "puedan leer en voz alta en un minuto" (SEP, 2011, p. 87) sea posible el logro de la capacidad. Para tal fin se requieren de distintas velocidades y niveles de atención para diferentes tipos de textos, de contenidos, de experiencia con los conceptos y los tipos de lenguaje. Así mismo, también resulta necesario localizar e integrar partes de un texto en una idea principal; realizar conexiones o comparaciones; identificar causas y efectos; integrar textos de formato distinto, como figuras, gráficas, tablas o mapas; todos ellos aprendizajes esperados correspondientes al nivel 3 de comprensión lectora del Programa para la Evaluación Internacional de Alumnos (PISA, por sus siglas en inglés) de la Organization for Economic Co-operation and Development (OECD) (SEP, 2011, p. 85).

Es indispensable que el estudiantado tenga la capacidad de "alfabetismo académico" el cual no se logra en la práctica educativa desde el paradigma tradicional (Caldera y Bermúdez, 2007; Díaz, 2005). Para favorecer las aproximaciones de los estudiantes a los avances de la ciencia y la tecnología, que en el caso de medicina es la base de un servicio de la mejor calidad posible (lo cual es un valor agregado a la atención de la salud), se necesita "el desarrollo de la habilidad lectora", ya que es "una de las claves para un buen 
aprendizaje en todas las áreas del conocimiento, dentro y fuera de la escuela" (SEP, 2011, p.87).

Literacidad, como también sucede con alfabetización, es un concepto polisémico. Por ello, se ha intentado acotar la capacidad en función del manejo de conceptos y estrategias complejas de lectura y escritura, de manera que Hernández (2012, p. 44) la convierte en palabra de dos términos y menciona que por literacidad académica "debe entenderse el conjunto de prácticas letradas que ocurren y tienen sentido dentro de una comunidad académica, discursiva y disciplinar." Si bien esto implica el conocimiento de un código disciplinar para saber qué decir —en el caso de la medicina para darle significado a los síntomas y signos de los pacientes - la competencia lingüística (leer y escribir de manera compleja) requiere, además, de la capacidad de saber "cómo decirlo de manera apropiada en cualquier situación dada" (Pérez, 2008, pp. 116-117) tanto para explicarle a los pacientes lo que significan sus signos y síntomas, describirlos en el expediente clínico en lenguaje técnico, como para "acceder a la información adecuada, interpretarla, juzgarla y aprovecharla para tomar decisiones bien fundamentadas" (Falcón y Luna, 2012, p. 92).

En consecuencia, estas capacidades deben ser parte del bagaje de los estudiantes que ingresan a la universidad, ya que son necesarias para construir conceptos científicos, filosóficos, sociales y culturales, de mayor complejidad (UNESCO, s.f.); sin embargo, en la realidad el énfasis en la conformación de las habilidades indispensables para convertir la información en conocimiento aplicable en una situación específica y resolver un problema no es lo habitual para los profesores de niveles previos al ingreso a la Universidad Juárez Autónoma de Tabasco.

\subsection{Experiencia previa del equipo de investigación}

El equipo de investigación ha desarrollado procesos de aula para facilitar la formación de las capacidades necesarias para que los estudiantes sean competentes en la gestión de recursos conceptuales y procedimentales para su aplicación en situaciones concretas de la vida real. De acuerdo con la línea de razonamiento de la AMFEM, "la gestión del conocimiento" - una de las capacidades de la literacidad (Caro y Arbeláez, 2009, p. 5) que debe tener quien egresa de las escuelas de medicina- es:

identificar las necesidades de conocimientos personales o grupales, proponiendo e instrumentando actividades formativas con objeto de favorecer la actualización e 
integración de comunidades de aprendizaje eficientes para contribuir a la mejora continua del desempeño del personal de salud y la población, y resolver los problemas de salud mediante la utilización del mejor conocimiento existente. (Abreu et al., 2008, p.18)

En experiencias anteriores en la Universidad de Tabasco, el equipo de investigación encontró, de manera empírica, que los estudiantes de semestres intermedios de la licenciatura de Medicina - a pesar de haber cursado metodología de la investigación, herramientas de la computación, salud pública, epidemiología, y otras como anatomía, que harían suponer de acuerdo a la lógica ordinaria de los cursos estructurados por asignaturas en bloques "básico" y "clínico"- los estudiantes y las estudiantes de semestres intermedios de la Licenciatura de Medicina carecen de una robusta estructura cognoscitiva para comprender la lectura. Además, halló debilidades para la elaboración de escritos, fuera en las formas de organizar ideas, jerarquizar conceptos o presentar resultados de sus observaciones en los campos clínicos. Al inicio de los cursos los estudiantes no mostraron capacidad para la búsqueda de información en bibliotecas virtuales, análisis de la información o realizar análisis de problemas (Muñoz y Maldonado, 2011).

Esta es la razón por la que el equipo de investigación se planteó como objetivo promover en los estudiantes la capacidad de identificar los problemas que debe resolver el médico en el primer nivel de atención, entendiendo por problema la situación de la que "no se tiene información suficiente para contestar una pregunta o cuando la información que se tienen es ambigua, incompleta, contradictoria o insuficiente para responder dicha pregunta" (Aguilar, 2011, diapositiva 3). De manera paralela, se trabajó en la formación de habilidades tendientes a la alfabetización como inicio al manejo de bases de datos y bibliotecas virtuales.

El tipo de escrito que se les pidió redactar en cursos anteriores, con el propósito de que hicieran diagnósticos contextuales, fue un "informe", ya que el concepto de "ensayo" tiene no sólo implicaciones menos definidas sino, además, diferentes connotaciones. Tanto en la plataforma, creada para estos fines, como a sus direcciones electrónicas se enviaron instrucciones y apoyos para que se realizaran actividades para su discusión en las sesiones presenciales.

Estos procesos han sufrido modificaciones y avances para centrarse en la elaboración de un informe escrito consistente en un diagnóstico del contexto. El instrumento para 
analizar en los cursos los niveles de la capacidad de construcción de un texto por los estudiantes y las estudiantes — producto observable de la comprensión lectora- desde una primera escritura, corrección, reescritura, nuevas correcciones, fue una escala de cuatro niveles que se construyó de acuerdo con Morales (2002). En esta escala los niveles son: 1) transcripción, reproducción o paráfrasis de un texto o con la sustitución de algunas palabras; 2) reproducción literal, parafraseo, tratamiento parcelado, no hay cierre; 3) persistencia del parafraseo, fallas en la vinculación entre los párrafos, y 4) construcción de un texto integrado donde hay párrafos de transición entre las temáticas, presencia de conectores, uso coherente de las referencias y se encuentra jerarquización de los conceptos. Para determinar los niveles de avance se identificaron palabras clave. Como ejemplo en la elaboración de un proyecto, los estudiantes que habían escrito inicialmente "transmitir" y "adiestrar", "diabético", las cambiaron por "construir", "educar", "paciente con diabetes" como parte de su proceso.

Sea porque se habían formado en ambientes donde la literacidad no era percibida como necesaria (la exposición en clase es explícita en los programas de estudio de medicina, hay periodos de tres semanas para exámenes al final de cada "ciclo largo" en el calendario escolar de la Universidad), porque los estándares que se plantearon eran más altos que los que se podía cumplir, o porque eran parte de una respuesta ante tareas diferentes; en cualquier caso el estudiantado mostró incomodidad, a pesar del apoyo facilitado para las experiencias anteriores y aún cuando se logró la elaboración tareas que requirieron de la lectura de varias fuentes y del procesamiento de la información (Muñoz y Maldonado, 2011; Muñoz, 2012; Muñoz, Maldonado y Bello, en prensa), la proporción de estudiantes que finalizó los cursos elaborados con ese propósito fue baja.

\subsection{Objetivo}

El objetivo de este estudio fue analizar los apoyos pedagógicos con que contaron los estudiantes de nuevo ingreso a medicina para construir conocimiento a partir de información que ha de leerse en diferentes textos y en diferentes formatos de acuerdo con las preguntas:

1) ¿Cuáles son las prácticas de lectura y escritura que realizan los estudiantes?

2) ¿Cómo es el acompañamiento de los docentes para la producción de escritos por los estudiantes?

3) ¿Cuál es el valor que los estudiantes les confieren a esas prácticas? 


\section{Material y método}

Se realizó una investigación cualitativa de estudio de caso (Hernández, et al., 2006, pp.223-224) de tipo de correlación de variables/prospectivo (Polit y Hungler, 1985, p. 160). La validez de construcción se obtuvo a partir del análisis, entre otros documentos de la administración, de las prácticas escolares a partir de la lectura del tipo de evaluaciones y características de la acreditación de los cursos, tanto en el plan de estudio como en los programas de asignatura, disponibles en: http://www.ujat.mx/interior.aspx?ID=69 (Fontoura et al., 2008, p. 219). Como se trató de un estudio de caso no se hizo la validación externa; a pesar de ello, los resultados de este estudio dieron origen a una base de datos como protocolo para la recolección de datos lo que permitió determinar la confiabilidad de la investigación, de acuerdo con Fontoura et al. (2008, p. 220).

Una categoría en este estudio fueron los escritos solicitados por el personal docente para la entrega de escritos y sus características. Otra fue el acompañamiento que, en forma de evaluación formativa, realizaron los docentes, ya que esta construye habilidades para que los estudiantes aprendan a aprender. Como definición de esta última se consideró la de la $\operatorname{OECD}(2005$, p. 1) que "consiste en evaluar el progreso y los conocimientos del alumno de forma frecuente e interactiva. De esta manera los maestros pueden ajustar sus programas para satisfacer mejor sus necesidades educativas". La otra categoría fue el sentido que para los estudiantes tiene el elaborar escritos.

\subsection{Participantes}

En el semestre en que se realizó la exploración hubo 350 estudiantes de nuevo ingreso a la Licenciatura de Medicina en agosto de 2011 en el campus principal de la Universidad de Tabasco, la cual es dependencia descentralizada del Estado. En la misma ciudad sede existe la posibilidad de ingresar a medicina en dos universidades privadas. Los estudiantes provinieron de las escuelas de nivel medio superior de todo el estado de Tabasco. Aún cuando no hay requisito mínimo de promedio de calificaciones, ya que la demanda para ingresar a este campus es alta, se acepta a los que obtienen los mayores puntajes en el examen de ingreso, los cuales se aplican y evalúan por el Centro Nacional de Evaluación para la Educación Superior, A.C. (CENEVAL). Se aplicaron los cuestionarios en los grupos de clase hasta saturar las categorías, cuando los nuevos datos no contribuyeron a 
desarrollar otras (Hernández et al., 2006, p. 563). Esto se observó una vez contestados los primeros 50, pero se continuó hasta abarcar una meta de 100 cuestionarios.

\subsection{Recolección de la información}

Para realizar el estudio se hizo un cuestionario con preguntas abiertas que se validó mediante la evaluación de tres docentes de otras dependencias educativas (Tabla 1). En el instrumento final, las preguntas 2, 3 y 5 se refieren a las prácticas que tienen los estudiantes para realizar sus escritos, las 1, 6 y 7 de las indicaciones y apoyos que reciben de los docentes, y 4, 8 y 9 acerca de las motivaciones que tienen para efectuar los escritos. Previo a la aplicación en agosto de 2011, en el verano de ese año se hizo un estudio piloto con estudiantes que acudieron a los cursos de "ciclo corto" que se llevan a cabo en la Universidad de Tabasco en cuatro semanas de junio y tres de agosto de cada año. Durante esta fase, los estudiantes son esencialmente los mismos de los cursos de "ciclo largo", de la última semana de agosto a enero del siguiente año y de febrero a junio, por lo que no se presentaron sesgos. Una vez obtenidas las respuestas se desarrollaron las categorías de análisis de cada una de las preguntas, de manera que se hizo una base de datos. Los cuestionarios se aplicaron y las respuestas se codificaron. Se identificaron palabras clave en las respuestas para el análisis de las variables.

\subsection{Consideraciones éticas}

A cada estudiante se le solicitó participar en el estudio. En caso de respuesta afirmativa se les solicitó que firmaran un consentimiento informado y se les explicó la posibilidad de no terminar el cuestionario a pesar del consentimiento. Cada cuestionario tuvo un número de registro sin posibilidad de identificación del participante. Estas actividades forman parte del proyecto de investigación "Aplicaciones de las neurociencias para el desarrollo de habilidades metacognitivas en la construcción de pensamiento sistémico y complejo", registrado en la Dirección de Investigación de la Universidad clave UJAT-DACS2011-21.

\section{Resultados y discusión}

Posterior a la aplicación de los cuestionarios, se analizó el vaciado en la base de datos (Tabla 2). Con el fin de cumplir con el primer objetivo, identificar las prácticas de literacidad 
de los estudiantes de nuevo ingreso a Medicina, se analizaron las respuestas de las preguntas 2, 3 y 5 . En este apartado se encontró que el tipo de escrito de mayor frecuencia que se les solicitó fue el ensayo, $57 \%$ de las veces, seguido por resúmenes en $27 \%$. Los estudiantes también consideraron como escritos los mapas mentales y los mapas conceptuales, y también mencionaron análisis y escritos temáticos como tareas solicitadas por sus docentes. Los estudiantes elaboraron sus escritos (pregunta 3 ) de acuerdo a criterios de formato (90\%), ejemplos: "buena ortografía, tamaño de letra adecuado, diseño de texto, extensión dependiendo del tipo de texto" (cuestionario 83), "algunos profesores piden una extensión de tres cuartillas, letra arial, tamaño 12" (cuestionario 50).

La mayor parte del estudiantado no tenía elaborada una estrategia (pregunta 5) definida para elaborar un escrito como con base en preguntas guía para, entre otros, elaborar una postura en una situación ambigua o resolver un problema. La respuesta con mayor frecuencia fue leer acerca del tema solicitado e identificar los conceptos que los estudiantes consideraban debía estar en su escrito en $68 \%$. Menos estudiantes, solo un $15 \%$, mencionaron que escribían después de la identificación de las ideas principales; y que hacían primero un borrador, lo revisaban y después lo reelaboraban un $13 \%$. Aunque $3 \%$ de ellos mencionaron contrastar la información, para $68 \%$ fue suficiente una fuente, independientemente de la confiabilidad del contenido. Ejemplos: "primero leer el tema indicado, subrayar, comprender lo que se subrayó, luego transcribirlo o escribir desde nuestro punto de vista" (cuestionario 21), "transcripción de textos" (cuestionario 76), y en ocasiones fue un capítulo de libro que sus profesores les indicaron tomar como fuente.

En este sentido los estudiantes no realizaron comentarios acerca de la dificultad que significó adentrarse en un proceso no solo de mayor complejidad conceptual e instrumental -se requieren las competencias necesarias para transformar la información en conocimiento-, sino que se configura como un campo de aplicación de la ciencia con un lenguaje disciplinar -en este caso la medicina- que le es propio (Peña, 2008). Ya que en su mayoría no hicieron mención a la internet (excepto cuestionarios 1 y 61), no describieron sus estrategias o dificultades para identificar fuentes de información, bases de datos, portales, bibliotecas virtuales de calidad, aunque algunos, por ejemplo, se quejaron del "exceso de información que hay acerca de un tema" (cuestionario 17).

Al analizar los resultados del acompañamiento del profesorado al grupo de estudiantes, meta del segundo objetivo, preguntas 1,6 y 7, se encontró que un dato de mayor constancia 
es la alta frecuencia con que los docentes solicitaron la elaboración de escritos para asignar una calificación por "participación en clase" (tal como está explícito en los programas de asignatura y el formato de planeación docente), con lo que pierde la oportunidad de fortalecer una "herramienta de pensamiento" (Peña, 2008 , p. 3). Aunque 22\% se refiere a "poco", 59\% respondió que la mayoría de sus profesores les solicitaban hacer escritos, incluso para la asignatura "morfología".

Aunque los estudiantes mencionan la realización de resúmenes (26\%) y mapas conceptuales $(2 \%)$, es notable la proporción de estudiantes que se refieren a la realización de ensayos (57\%). Es posible que la solicitud de tal número de ensayos se debió a la falta de claridad de las características de estructura e intención de lo que es un ensayo (difícil de escribir en una semana), como posiblemente sucede en los resultados de Montemayor (2006). Esto también hizo evidente la falta de claridad de los docentes de lo que es construir la competencia comunicativa, precisamente porque no se proporcionaron, a los estudiantes los criterios para la elaboración de los escritos. De acuerdo con Peña (2008, p. 8), éste es un obstáculo para una comunicación con "objeto del saber-cómo se aprende", problema no resuelto que se comparte con docentes de nivel superior de otros países de América (Hernández, 2000; Morales, 2004).

El que los docentes asignaron calificaciones a sus escritos predominantemente por ortografía y gramática, ejemplos: "por cada palabra mal escrita bajan un punto" (cuestionario 65), y que por escribir con faltas ortográficas "te humillan y te dicen cosas" (cuestionario 56), refleja un limitado criterio para evaluar la escritura. La ausencia de una verdadera retroalimentación, o de algún otro tipo de acompañamiento a los grupos de estudiantes, tal como se encuentra en las respuestas a las preguntas 6 y 7 , pone de manifiesto que estas actividades tienen poco valor formativo, cuando, de acuerdo con Pérez (2008), debería ser evidente la necesidad del acompañamiento a los estudiantes para brindarles herramientas con la finalidad de aumentar los estándares de lectura y escritura.

Planes de estudio estructurados en bloques básicos y clínicos, con persistencia de asignaturas canónicas que solo se aprenden repitiendo para memorizar, como es anatomía, histología, incluso bioquímica o biología molecular desde la estructura el paradigma de la escuela tradicional, evita que se genere interés, tanto en los directivos de muchas de las universidades de América Latina Universidades como en muchos de los docentes y por supuesto en los estudiantes, por el desarrollo de actividades innovadoras de aula o de 
ambientes virtuales, sobre todo si es difícil demostrar el desempeño, mientras que el aprovechamiento se hace claramente con repetición de definiciones. La actuación con base en un diseño curricular centrado en contenidos enciclopédicos al mismo que una débil conceptuación del contenido (Díaz, 2006, p. 594), más que en un genuino interés académico, es lo que sabotea las modificaciones que se pretenden realizar en las instituciones educativas. En este sentido, los estudiantes mencionaron que escribieron con base en un texto indicado por sus profesores, ejemplo: "documento o material que se nos da" (cuestionario 66), esto no los condujo a una lectura y escritura más "extensiva" ni más “intensiva”, lo cual es necesario para "comparar y valorar críticamente" (Peña, 2008, p. 3).

Para realizar la valoración de los estudiantes acerca de las prácticas de lectura y escritura, y la motivación que tuvieron para hacerlo, se analizaron las respuestas de las preguntas 4, 8 y 9. Hubo diferencias en las respuestas a la pregunta 4 en relación con la pregunta 9. Aunque elaboradas en términos muy semejantes, para los estudiantes hubo diferencia entre acreditar los cursos (la escuela) y aprender medicina (el hospital). La pregunta 4 estaba a continuación de preguntas relacionadas con lo que los profesores solicitan y la 9 incluye "útil” y "médico".

La principal respuesta a "para qué elabora sus escritos en la escuela" fue aprender conceptos (54\%). Ejemplos: "tener mayor entendimiento" (cuestionario 27), "comprender mejor el tema" (cuestionario 45), "me doy cuenta de que ya aprendí" (cuestionario 88). Para lo que los estudiantes consideraron el contexto de aula, escribir no fue un ejercicio posterior a una lectura crítica -a partir de varias fuentes-, sino como ejercicio nemotécnico, ejemplo: para "describir lo aprendido en clases" (cuestionario 32). Otra razón fue aumentar su habilidad de escritura (20\%), "mejorar nuestras formas de redactar textos" (cuestionario 21), "mejorar nuestras habilidades de redacción" (cuestionario 46). Allí faltó la escritura como actividad de comunicación y emitir mensajes bien construidos. Para otros, escribir era tangencial a la identificación de información (16\%), pues de esta manera podían "tener más información" (cuestionarios 51, 84). Sólo 4 respondieron que era útil para "emitir una opinión" (cuestionario 35).

Estas respuestas difirieron de las de la pregunta 9, acerca de la "utilidad de la escritura" en la vida profesional. Aunque 40\% mantienen la utilidad en "aprender conceptos", $29 \%$ se refirieron a la necesidad de elaborar opiniones informadas, ejemplos: "encontrar datos relevantes" (cuestionario 1), "me despierta el interés de investigar y conocer nuevos 
temas" (cuestionario 62), "nos ayuda a estar más informados y actualizados" (cuestionario 64), "me permiten expresar mis conocimientos" (cuestionario 73 ), y $24 \%$ a que la práctica de la escritura es una necesidad académica, "cuando me toque elaborar proyectos clínicos o (describir) casos clínicos" (cuestionario 66), "necesitamos transmitir ideas, emociones y conocimientos de todas las formas posibles y como método de estudio" (cuestionario 89). También en esta pregunta hubo un $3 \%$ que respondió su descontento a escribir, ejemplo: "ninguna utilidad, hay otros métodos mejores" para aprender (cuestionario 43).

\section{Conclusiones}

Como toda investigación de asociación la limitante de este estudio es que no se clarifican las relaciones de causa a efecto. Con el riesgo de sobreinterpretar las relaciones entre las categorías se ha tratado de explicar hechos que se han observado en otros grupos acerca de la literacidad, lo cual constituye una debilidad, ya que los participantes en esta investigación no necesariamente fueron semejantes a los grupos con los que el equipo de investigación había trabajado anteriormente, aún cuando haya sido en la misma escuela de medicina. A pesar de las limitantes del estudio se encontró:

1. El profesorado solicitó a los grupos de estudiantes un alto volumen de trabajos escritos que difícilmente se realizaron de acuerdo al propósito de formarles en habilidades de la literacidad.

2. Comprender lo que se lee para elaborar escritos es un proceso que se aprende con la práctica. Si como sucede en este caso, donde no se acompañó de retroalimentación para la mejorarlos, es poco probable que se egrese con formación para la gestión del conocimiento. La construcción de la literacidad requiere de planeación estratégica y logro de metas (Salgado, 2012), a partir de un cuidadoso trabajo docente.

3. Los estudiantes consideran dos tipos de escritos, los que se hacen para los cursos y los que se harán al egreso. Esta desconexión entre la escuela y la práctica profesional posiblemente dependa del tipo de planes de estudio y de los programas de asignatura, elaborados desde el paradigma de la escuela tradicional. Hace falta repensar la práctica docente para que, a su vez, los estudiantes resignifiquen las tareas escolares como metas para el logro de la capacidad del médico.

4. Los estudiantes y las estudiantes, como tampoco el personal docente, son homogéneos, por lo que los programas y las actividades de clase deben responder a la 
heterogeneidad (Kalman, 2008). Debe respetarse el derecho a las diferencias en el sentido de que solo $18 \%$ manifiestan facilidades para escribir. Independientemente de la calidad de sus escritos, ellos se sienten cómodos escribiendo. Hacen falta ayudas específicas para los demás estudiantes que pueden tener modos distintos en cómo aprenden mejor.

5. Escribir no es fácil, y menos en las condiciones en las que lo realizaron estos estudiantes, sin criterios para la elaboración ni ayudas en la búsqueda y manejo de la información. La mayor dificultad que tienen es la identificación de conceptos que deben estar en los escritos (35\%), ejemplo: "encontrar las palabras exactas y correctas" (cuestionario 57). Se debe a que los estudiantes dependen de "saber qué es lo que el profesor quiere realmente" (cuestionario 96), pero que probablemente tampoco tiene definido su profesor.

6. En este estudio se analizaron solo de manera tangencial las dificultades para la gestión de recursos en la red. Superar los problemas para "la gestión ágil y económica del conocimiento" (Caro y Arbeláez, 2009, p 20) disponible en la red, lleva a considerar los ejes de próximas intervenciones sugeridos por esas autoras: uso y creación de hipertextos, uso de la interactividad e interlocución de índole académica, entre otros, que propicien la literacidad en el ámbito electrónico. 


\section{Referencias}

Abreu Hernández, Luis Felipe; Cid García, Ángel Neftalí; Herrera Correa, Gloria; Lara Vélez, Víctor Manuel; Laviada Delgadillo, Rocío; Rodríguez Arroyo, Christian y Sánchez Aparicio, Juan Antelmo. (2008). Perfil por competencias del médico general mexicano. México: Asociación Mexicana de Facultades y Escuelas de Medicina. Recuperado de: http://www.amfem.edu.mx/intranet/descargas/competencias.pdf

Aguilar Morales, Jorge Everardo. (2011) ¿Cómo seleccionar un problema de investigación? México: Asociación Oaxaqueña de Psicología A. C. Recuperado de: http://www.conductitlan.net/

Caldera, Reina y Bermúdez, Alexis. (2007). Alfabetización académica: comprensión y producción de textos. Educere, 11(37), 247-255. Recuperado de: http://www.saber.ula.ve/bitstream/123456789/20149/2/articulo9.pdf

Caro Salcedo, Laura Camila y Arbeláez Echeverri, Nora Cristina. (2009). Hipertextualidad, literacidad y discurso académico: conceptos para la gestión del conocimiento en la red. Revista Virtual Universidad Católica del Norte, 28. Recuperado de: http://revistavirtual.ucn.edu.co/index.php/RevistaUCN/article/view/85/175

Cassany, Daniel y Morales, Oscar Alberto. (2008). Leer y escribir en la universidad: hacia la lectura y la escritura crítica de géneros científicos. Memoralia, 5(2), 69-82. Recuperado de: http://lenguharas.jimdo.com/memoralia/

Centres for Disease Control and Prevention. (SF). Policy impact: prescription painkiller overdoses. CDC. Recuperado de: http://www.cdc.gov/homeandrecreationalsafety/rxbrief/index.html

Committee on Developments in the Science of Learning. (2000). How people learn. Brain, mind, experience, and school. Washington: The National Academies Press. Recuperado de: http://www.nap.edu/openbook.php?isbn=0309070368

Díaz Barriga, Ángel. (2005). El enfoque de competencias en la educación ¿Una alternativa o un disfraz de cambio? Perfiles Educativos, 28(111):7-36.

Díaz Barriga, Ángel. (2006). Las pruebas masivas: análisis de sus diferencias técnicas. Rev Mex Invest Educ, 29(11), 538-615. Recuperado de: http://redalyc.uaemex.mx/pdf/140/14002912.pdf

Falcón, María y Luna Ruiz-Cabello, Aurelio. (2012). Alfabetización en salud; concepto y dimensiones. Proyecto europeo de alfabetización en salud. Rev Comunicación Salud, 2(2), 91-98. Recuperado de: http://www.revistadecomunicacionysalud.org/index.php/rcys/article/view/35/42

Fontoura Cartana, Maria do Horto; De Souza, Maria de Lourdes.; Schmidt Reibnitz, Kenya y Alonso Castillo, María Magdalena. (2008). El estudio de caso en investigación en enfermería. En: Investigación cualitativa en enfermería: contexto y bases 
conceptuales. Do Prado Marta Lenise; De Souza, Maria de Lourdes y Carraro, Telma Elisa. edits. (pp. 213-222). Washingrton: OPS.

Hernández, M. Rosario. (2000). La evaluación como estrategia docente en el desarrollo de la competencia escritora en E/LE. ASELE, Actas, 11, 431-438. Recuperado de: http://cvc.cervantes.es/ensenanza/biblioteca ele/asele/pdf/11/11 0431.pdf

Hernández Rojas, Gerardo. (2008). Teorías implícitas de lectura y conocimiento metatextual en estudiantes de secundaria, bachillerato y educación superior. Rev Mex Invest Educ, 13(38), 737-771. Recuperado de: http://redalyc.uaemex.mx/pdf/140/14003804.pdf

Hernández Rojas, Gerardo. (2012). Teorías implícitas de escritura en estudiantes pertenecientes a dos comunidades académicas distintas. Perfiles Educativos, 34(136): $42-62$.

Hernández Sampieri, Roberto; Fernández Collado, Carlos y Baptista Lucio, Pilar. (2006). Metodología de la investigación. México: McGraw-Hill.

Institute of Medicine of the National Academies. (2012). Best care at lower cost: the path to continuously learning health care in America. Recuperado de: http://www.iom.edu/ /media/Files/Report\%20Files/2012/Best-

Care/Best\%20Care\%20at\%20Lower\%20Cost Recs.pdf

Kalman, Judith. (2008). Discusiones conceptuales en el campo de la cultura escrita. Rev Iberoamericana Educación, 46, 107-134. Recuperado de: http://www.rieoei.org/rie46a06.htm

Morales Ardaya, Francisco. (2004). Evaluar la escritura, sí... Pero ¿Qué y cómo evaluar? Acción Pedagógica, 13(1), 38-49. Recuperado de: http://www.saber.ula.ve/bitstream/123456789/17141/2/articulo4.pdf

Morales, Oscar. (2002). ¿Cómo contribuir con el desarrollo de las competencias de los estudiantes universitarios como productores de textos? Educere, vol. 5, núm. 16, pp. 385-389. Recuperado de: http://www.saber.ula.ve/bitstream/123456789/19671/1/articulo1.pdf

Muñoz Cano, Juan Manuel. (2012). Experiencia metacompleja para la construcción de un modelo de diagnóstico médico por estudiantes. Educ Med, 15(2): 89-94. Recuperado de: http://scielo.isciii.es/pdf/edu/v15n2/original1.pdf

Muñoz Cano, Juan Manuel y Maldonado Salazar, Teresita. (2011). Aprendizaje con base en proyectos para desarrollar capacidades de problematización en educación superior. Actualidades Investigativas en Educación, 11(1). Recuperado de: http://redalyc.uaemex.mx/src/inicio/ArtPdfRed.jsp?iCve $=44718060014$

Muñoz Cano, Juan Manuel; Maldonado Salazar, Teresita y Bello, Juan. (En prensa) Desarrollo de proyectos para la formación de la competencia intercultural por estudiantes de Medicina. Educ Med. 
Organización Mundial de la Salud. (2008). World Alliance for Patient Safety. Forward Programme 2008-2009. Ginebra: WHO. Recuperado de: http://www.who.int/patientsafety/information centre/reports/Alliance Forward Program me 2008.pdf

Organisation for Economic Co-operation and Development. (2005). Formative assessment: improving learning in secondary classrooms. Recuperado de: http://www.oecd.org/edu/ceri/35661078.pdf

Pérez, Glenys. (2008). Las producciones textuales de los estudiantes en el contexto universitario. Memoralia, 5(3), 109-122. Recuperado de: http://lenguharas.jimdo.com/memoralia/

Peña Borrego, Luis Bernardo. (2008). La competencia oral y escrita en la educación superior. Ministerio de Educación de Colombia. Recuperado de: http://www.mineducacion.gov.co/1621/articles-189357 archivo pdf comunicacion.pdf

Polit, Denise F. y Hungler, Bernardette P. (1985). Investigación científica en ciencias de la salud. México, D.F.: Interamericana.

Salgado García, Edgar. (2012). Enseñanza para la comprensión en la educación superior: la experiencia de una universidad costarricense. Rev Iberoamer Educ Sup, 3(8), 34-50. Recuperado de: http://ries.universia.net/index.php/ries/article/view/157/pdf 28

Secretaría de Educación Pública. (2011). Plan de estudios 2011. Educación básica. México: Secretaría de Educación Pública. Recuperado de: http://basica.sep.gob.mx/dgdc/sitio/pdf/PlanEdu2011.pdf

Starfield, Barbara. (2000). Is US Health Really the Best in the World? JAMA, 284(4), 483-485. doi: 10-1001/pubs.JAMA-ISSN-0098-7484-284-4-jco00061

United Nations Educational, Scientific and Cultural Organization. (SF). LAMP - Literacy Assessment and Monitoring Programme. Recuperado de: http://www.uis.unesco.org/literacy/Pages/lamp-literacy-assessment.aspx

United Nations Educational, Scientific and Cultural Organization. (2009). La nueva dinámica de la educación superior y la investigación para el cambio social y el desarrollo. Sede de la UNESCO, Comunicado de la Conferencia Mundial sobre la Educación Superior, París, 5 al 8 de julio. Comunicado del 8 de julio de 2009. Recuperado de: www.unesco.org/education/WCHE2009/comunicado es.pdf

Warner, Margaret; Chen, Li Hui y Makuc, Diane M. (2009). Increase in fatal poisonings involving opioid analgesics in the United States, 1999-2006. NCHS Data Brief. Recuperado de: http://www.cdc.gov/nchs/data/databriefs/db22.htm 


\section{Tabla 1}

\section{Cuestionario para los estudiantes}

- ¿ ¿Con qué frecuencia le solicitan sus profesores que elabore escritos como parte del trabajo escolar?

- ¿Qué tipo de escritos les solicitan?

- ¿ ¿Qué características deben tener los escritos? (extensión y estructura).

- ¿Para qué elabora los escritos? ¿le sirve de algo como estudiante?

- ¿Cómo elabora sus escritos? Por favor indíquenos el procedimiento que sigue para escribirlos.

- ¿Sus profesores revisan sus escritos? ¿Le hacen sugerencias? Si es así ¿le solicitan que reelabore el escrito y lo entregue con los cambios?

- ¿ ¿Cómo evalúan los profesores los escritos que elabora?

- ¿Cuáles son las principales dificultades que enfrenta para elaborar escritos?

- ¿Elaborar escritos es útil?, ¿por qué?, ¿sirve para su formación como médico?, ¿cómo? Por favor sustente si respuesta.

Fuente: Elaboración de los autores. 
Tabla 2

Frecuencias

\begin{tabular}{|c|c|c|c|c|c|}
\hline Categorías & \multicolumn{5}{|c|}{ Codificación (\%) } \\
\hline \multirow{2}{*}{$\begin{array}{l}\text { 1. Frecuencia de } \\
\text { la solicitud }\end{array}$} & $\begin{array}{c}\text { Sin } \\
\text { respuesta }\end{array}$ & Pocos & La mayoría & Casi todos & \\
\hline & 1 & 22 & 59 & 18 & \\
\hline \multirow[b]{2}{*}{ 2. Tipo de escrito } & Ensayo & Análisis & Resúmenes & Mapas & Temáticos \\
\hline & 57 & 9 & 26 & 2 & 6 \\
\hline \multirow{2}{*}{$\begin{array}{l}\text { 3. Características } \\
\text { principales }\end{array}$} & Formato & $\begin{array}{l}\text { Conceptos } \\
\text { temáticos }\end{array}$ & $\begin{array}{c}\text { Sin } \\
\text { respuesta }\end{array}$ & & \\
\hline & 90 & 9 & 1 & & \\
\hline \multirow{2}{*}{$\begin{array}{l}\text { 4. Para qué } \\
\text { escribir }\end{array}$} & $\begin{array}{l}\text { Aprender } \\
\text { conceptos }\end{array}$ & $\begin{array}{l}\text { Mejorar } \\
\text { escritura }\end{array}$ & $\begin{array}{c}\text { Identificar } \\
\text { información }\end{array}$ & Obligación & $\begin{array}{l}\text { Opiniones } \\
\text { informadas }\end{array}$ \\
\hline & 54 & 20 & 16 & 6 & 4 \\
\hline \multirow{2}{*}{$\begin{array}{l}\text { 5. Estrategia para } \\
\text { escribir }\end{array}$} & $\begin{array}{l}\text { Seleccionar, } \\
\text { escribir }\end{array}$ & $\begin{array}{l}\text { Listado de } \\
\text { conceptos }\end{array}$ & $\begin{array}{c}\text { Hacer } \\
\text { borradores }\end{array}$ & $\begin{array}{c}\text { Contrastar } \\
\text { información }\end{array}$ & $\begin{array}{c}\text { Sin } \\
\text { respuesta }\end{array}$ \\
\hline & 68 & 15 & 13 & 3 & 1 \\
\hline \multirow{2}{*}{$\begin{array}{l}\text { 6. Retro- } \\
\text { alimentación }\end{array}$} & No la define & $\begin{array}{l}\text { Conceptos } \\
\text { temáticos }\end{array}$ & $\begin{array}{c}\text { Ortografía y } \\
\text { gramática }\end{array}$ & Congruencia & $\begin{array}{c}\text { Sin } \\
\text { respuesta }\end{array}$ \\
\hline & 72 & 18 & 6 & 3 & 1 \\
\hline \multirow{2}{*}{$\begin{array}{l}\text { 7. Criterios de } \\
\text { evaluación }\end{array}$} & $\begin{array}{l}\text { No los } \\
\text { conoce }\end{array}$ & $\begin{array}{l}\text { Ortografía y } \\
\text { gramática }\end{array}$ & Congruencia & $\begin{array}{l}\text { Conceptos } \\
\text { temáticos }\end{array}$ & $\begin{array}{c}\text { Sin } \\
\text { respuesta }\end{array}$ \\
\hline & 54 & 30 & 9 & 4 & 3 \\
\hline \multirow{2}{*}{$\begin{array}{l}\text { 8. Dificultades } \\
\text { para escribir }\end{array}$} & No tiene & $\begin{array}{l}\text { Identificar } \\
\text { conceptos }\end{array}$ & $\begin{array}{c}\text { Falta de } \\
\text { tiempo }\end{array}$ & $\begin{array}{l}\text { Construir } \\
\text { escritos }\end{array}$ & $\begin{array}{c}\text { Trabajo en } \\
\text { equipo }\end{array}$ \\
\hline & 18 & 53 & 16 & 12 & 1 \\
\hline \multirow{2}{*}{$\begin{array}{l}\text { 9. Utilidad de } \\
\text { escribir }\end{array}$} & $\begin{array}{l}\text { Aprender } \\
\text { conceptos }\end{array}$ & $\begin{array}{l}\text { Opiniones } \\
\text { informadas }\end{array}$ & $\begin{array}{c}\text { Práctica de } \\
\text { escritura }\end{array}$ & $\begin{array}{c}\text { Mejorar } \\
\text { ortografía }\end{array}$ & Ninguna \\
\hline & 40 & 29 & 24 & 4 & 3 \\
\hline
\end{tabular}

Fuente: Elaboración de los autores. 